\title{
Transformations agricoles et agricultures familiales : quelques défis mis en lumière durant l'Année internationale de l'agriculture familiale
}

\author{
Jean-Michel Sourisseau ${ }^{1 \mathrm{a}}$ \\ Marie-Aude Even ${ }^{2 a}$ \\ ${ }^{1}$ CIRAD \\ UMR ART-DEV \\ TA C- $113 / 15$ \\ 73, rue Jean-François Breton \\ 34398 Montpellier Cedex 5 \\ France \\ $<$ jean-michel.sourisseau@cirad.fr> \\ ${ }^{2}$ World Agricultures Watch initiative \\ Land and Water Division \\ Food and Agriculture Organization of the \\ United Nations (FAO) \\ Viale delle terme di Caracalla \\ 00153 Rome \\ Italie \\ $<$ MarieAude.Even@fao.org>
}

\footnotetext{
${ }^{a}$ Les opinions exprimées dans ce produit d'information sont celles du/des auteur(s) et ne reflètent pas nécessairement les vues ou les politiques de la FAO et des institutions soutenant l'initiative WAW.
}

doi: 10.1684/agr.2015.0764
E n choisissant de déclarer 2014 "Année internationale de l'agriculture familiale" (AIAF), les Nations Unies et les acteurs politiques et de la société civile ayant fait campagne pour ce choix invitaient le monde à s'interroger sur les défis rencontrés par les modèles et les formes de production agricole et leurs transformations rapides. L'objectif politique des promoteurs de l'année internationale était de montrer aux décideurs nationaux l'intérêt des formes familiales, implicitement par rapport aux formes non familiales - et en particulier l'agrobusiness dans des schémas de plus en plus industriels dont l'hégémonie, annoncée par les processus d'accaparement foncier et de financiarisation de l'agriculture, serait préjudiciable à la planète.

Le terme " agriculture familiale " n'est pas neutre. Il était jusque-là peu présent dans les textes et déclarations des institutions internationales du développement, ces dernières utilisant davantage la notion de "petite agriculture " pour cibler les formes agricoles les plus vulnérables. De fait, la catégorie "agriculture familiale" n'est pas une catégorie statistique et il est aujourd'hui impossible, avec les données disponibles, d'en mesurer précisément les contributions économiques et sociales. Les promoteurs de l'AIAF ont ainsi tenté de modifier les représentations en changeant de vocable. Ils ont en particulier loué le caractère inclusif du concept d'agriculture familiale et sa capacité à rassembler, au Nord comme au Sud, toutes les forces contre les formes les plus radicales d'agrobusiness et les tendances à la concentration et à l'industrialisation de l'agriculture. En continuité d'une dynamique inclusive, l'Organisation des Nations Unies pour l'alimentation et l'agriculture (FAO) a proposé des principes communs permettant une définition large et intégratrice de l'agriculture familiale, qui a été adoptée par le comité directeur international de l'AIAF qui rassemblait des représentants des gouvernements, de la société civile et d'agences de développement : "l'agriculture familiale est un mode d'organisation dans lequel la production agricole, forestière, halieutique, pastorale et aquacole est gérée et exploitée par une famille et repose principalement sur la main-d'oeuvre familiale, à la fois féminine et masculine. La famille et l'exploitation sont souvent liées, évoluent ensemble, et remplissent des fonctions économiques, environnementales, sociales et culturelles" (FAO, 2014). En insistant sur les liens entre les sphères productive et domestique, elle met en avant le fait que les agriculteurs familiaux sont d'abord et avant tout des acteurs sociaux, puis des producteurs, et qu'ils ne sauraient se voir réduits au statut de "fournisseurs de matières premières". Cette définition peut cependant paraître 
floue et certains syndicats, organisations de producteurs et représentants de la société civile jugent le terme trop englobant et pouvant désigner des exploitations engagées dans des processus de modernisation et d'artificialisation des systèmes de production, qu'ils jugent difficilement compatibles avec la promotion d'une agriculture plus respectueuse des hommes et de l'environnement. Ces acteurs préfèrent la référence au "paysan" et à ses valeurs. À l'opposé, d'autres trouvent les limites imposées vis-à-vis du travail salarié trop contraignantes. Finalement, d'autres soulignent que cette définition reste floue et qu'une définition plus opérationnelle et statistique est nécessaire, avec des critères disponibles dans les statistiques publiques et des seuils plus stricts, par exemple en termes de limite ou d'absence de main-d'œuvre salariée permanente. L'attention portée durant l'AIAF à la définition de l'agriculture familiale et des autres formes de production renvoie ainsi à des débats sur les représentations de l'agriculture, sur son avenir et sur son rôle dans les trajectoires nationales de développement. Elle est aussi liée aux interrogations sur les transformations rapides que connaît l'agriculture depuis les années 1960, sur les limites des modèles actuellement mis en oeuvre au Nord comme au Sud, et sur les inflexions souhaitables à y apporter.

La recherche a un rôle important dans ces débats et a été sollicitée durant l'AIAF. Elle apporte des éclairages sur la compréhension de ce qui se noue à l'intérieur des familles agricoles, en développant des méthodes d'investigation et de mesure originales et adaptées aux réalités des agricultures du monde d'aujourd'hui. Elle peut ainsi permettre, sur des critères objectifs et pertinents, de caractériser, mesurer et suivre l'évolution et les performances des différentes formes de production par des dispositifs d'études, d'observatoires, mais aussi via des statistiques nationales capables de les identifier. Mieux définir les agricultures familiales comme les autres formes de production est dès lors une base nécessaire pour construire des politiques publiques adaptées, mais aussi pour améliorer le ciblage de la mise en ouvre de ces politiques. Cet effort de définition et de construction d'une catégorie socio- politique repérable a fait l'objet de nombreux travaux et recommandations durant l'AIAF, mais sans que se dégage un consensus. En effet, ces travaux soulèvent des difficultés spécifiques au vu de la diversité des acteurs, des contextes, des hétérogénéités existant au sein de ces agricultures familiales, des données et variables disponibles, ainsi que des divers objectifs associés à ces définitions. De nombreux critères ont été proposés et discutés pour caractériser et différencier les formes de production : part du travail familial mobilisé dans la production agricole, nature des liens entre sphère productive et domestique, taille des exploitations, degré et processus d'insertion aux marchés, propriété des moyens de production, lieu de résidence des exploitants, dépendance par rapport aux acteurs de l'amont et de l'aval des filières, degré de spécialisation et de pluriactivité, ancrage dans sa communauté rurale et modalités de gestion des exploitations. Les travaux ont également soulevé la nécessité de proposer des typologies de ces agricultures familiales permettant de mieux saisir leur diversité. Pour autant, nombre de ces critères sont difficilement quantifiables et ne pourront pas être renseignés dans les statistiques nationales et internationales. L'initiative d'Observatoire des agricultures du monde (WAW - http://www.worldagricultureswatch.org/) a commencé à travailler sur ces questions dans le cadre d'un partenariat entre la FAO, le Fonds international pour le développement agricole (FIDA), le Centre de coopération internationale en recherche agronomique pour le développement (CIRAD) et des institutions et experts de sept pays. Un groupe international a été mis en place durant l'AIAF sur ce sujet. La FAO, en collaboration avec WAW et au sein de la stratégie globale des statistiques agricoles et rurales, va continuer à animer la réflexion, en croisant exigences statistiques dans le cadre de la refonte envisagée des données agricoles à l'échelle mondiale, et besoin d'approches compréhensives, incluant les points de vue de différents acteurs, et locales, pour contribuer effectivement à la décision politique.

Conjointement, dans le prolongement des débats de l'AIAF et des alertes sur les accaparements fonciers et la finan- ciarisation de l'agriculture lancées depuis la crise alimentaire de 2008, la recherche est sollicitée par la société civile pour mieux documenter et suivre les transformations à l'œuvre dans l'agriculture, en insistant sur les forces, mais aussi les faiblesses de l'agriculture familiale et des autres formes de production (Sourisseau et al., 2015). Il semble aussi que la recherche puisse contribuer à juger des risques comme des perspectives de la coexistence des différents modes de production, de leurs dynamiques et de la diversité des trajectoires de transformation possibles, et donc à fournir des connaissances objectives permettant de faciliter la décision politique. En effet, le rôle de la puissance publique, comme acteur volontaire impulsant les changements ou comme régulateur agissant, est désormais à nouveau reconnu comme un déterminant essentiel des processus de développement. Cependant, dans le même temps, la convergence des formes de production agricole et leur sélection par leur compétitivité semblent s'imposer comme des fatalités.

Ce numéro thématique des Cabiers Agricultures apporte une contribution à ces perspectives post-AIAF, en continuité de travaux initiés dès 2008 par l'initiative d'Observatoire des agricultures du monde et d'un séminaire international organisé sur ce sujet lors du $10^{\mathrm{e}}$ symposium de l'International Farming System Association (http:// www.worldagricultureswatch.org/events/ july-2012-10th-ifsa-symposium), portant sur les enjeux des agricultures familiales dans un contexte de pression de modernisation et de transformations structurelles.

Les processus de changement sont trop souvent présentés de façon désincarnée, comme des dynamiques génériques quasiment naturelles et dont les impacts macroéconomiques mesurés positivement justifieraient de passer outre les résistances locales à leur réalisation. Il s'agit au contraire ici, sur la base de données originales et dans des contextes contrastés, d'envisager la diversité des processus mondiaux et locaux de transformation des agricultures, le poids des politiques publiques dans ces processus, et leurs impacts sur les agricultures familiales prises dans leur diversité. Le numéro comprend un premier article de synthèse (Bosc et Bélières, 2015), qui met en regard les approches 
globales et macroéconomiques avec les dimensions et caractéristiques microéconomiques, à l'échelle des exploitations, pour comprendre la nature et la diversité des transformations de l'agriculture. La confrontation des deux échelles - macroéconomique et locale - alerte sur les risques de visions stéréotypées. La réalité des ménages et des exploitations agricoles nous montre un tout autre visage des transformations structurelles que la lecture des agrégats nationaux.

Les deux premières études de cas se focalisent sur deux facteurs emblématiques et largement médiatisés de changement: l'urbanisation (Parrot, $2015)$ et les accaparements fonciers (Burnod et Medernach, 2015). Parrot donne une image diachronique de la transformation d'une petite ville au Cameroun. Il insiste sur la singularité du processus urbain - singularité qui renvoie à la diversité des formes urbaines et de leurs impacts - et discute de ses risques et de ses opportunités pour les agricultures familiales. Burnod et Medernach bousculent nos présupposés sur les achats de terre à grande échelle. Leur étude fine de la coexistence entre petites agricultures familiales et agrobusiness à Madagascar nous montre qu'il n'y a pas de fatalité : les formes entrepreneuriales peinent finalement à se déployer, tandis que les relations entre agrobusiness et agricultures familiales ne sont pas nécessairement conflictuelles ni totalement en défaveur des secondes.

Les articles suivants témoignent des ambivalences entre les volontés nationales d'orienter les structures et logiques agricoles et les résistances ou adaptations des agricultures ciblées par ces transformations. Les injonctions nationales sont majoritairement productivistes; l'augmentation de la taille des exploitations et de leur degré de spécialisation dans des logiques d'intégration aux marchés ont été et restent encore largement les mots clés du développement agricole, tandis que la petite agriculture familiale, par ailleurs mal définie, est stigmatisée pour sa faible productivité. Les études de cas au Canada (Machum, 2015) et en Sierra Leone (Saravia-Matus et Gomez y Paloma, 2015) illustrent comment de telles politiques publiques, parce qu'elles sous-estiment les implications pour les familles et les territoires concernés, rencontrent des résistances qui elles-mêmes orientent vers de nouvelles trajectoires de changement. Ces articles montrent également comment la production statistique déforme parfois les réalités de terrain en sous-estimant notamment le secteur non marchand et les exploitations les plus petites.

L'étude de cas en Sierra Leone insiste aussi sur l'apport de modèles d'analyse politique et économique prenant en compte la diversité des types d'exploitation familiale et de leur orientation technico-économique.

Ces injonctions peuvent aussi renvoyer à des enjeux environnementaux normatifs, qui entrent le plus souvent en conflit avec les préconisations antérieures. Les représentations des éleveurs de l'Amazonie brésilienne (Cialdella, 2015) sur la réduction de la déforestation, et sur les nécessaires adaptations techniques induites, illustrent tout à la fois les capacités d'évolution rapide des systèmes familiaux et non familiaux, mais aussi une certaine inertie liée aux implications sociales (et souvent risquées) des changements techniques souhaités par les politiques nationales et internationales.

Les articles illustrent une diversité de trajectoires de transformation d'agricultures elles-mêmes diverses et opérant dans des contextes variés. Ils montrent aussi le rôle des politiques dans l'orientation de ces trajectoires. Différentes stratégies d'adaptation d'acteurs individuels et collectifs mettant en ouvre différentes formes d'agricultures y sont décrites, montrant ainsi la valeur ajoutée de travaux prenant en compte à la fois les dynamiques de transformation et la diversité des contextes et des formes agricoles coexistantes sur un même territoire. L'analyse des caractéristiques de cette diversité et des points de vue des différents types d'acteurs apparaît fondamentale pour expliquer ces stratégies, pour mieux comprendre les enjeux relatifs à la mise en ouvre des politiques et interventions techniques préconisées, et pour raisonner la participation des acteurs au dialogue sur les politiques agricoles.

\section{Références}

Bosc PM, Bélières JF, 2015. Transformations agricoles : un point de vue renouvelé par une mise en perspective d'approches macro et microéconomiques. Cahiers Agricultures 24:206-14. doi: 10.1684/agr.2015.0762

Burnod P, Medernach K, 2015. Interrelations entre agro-industrie et agricultures familiales vues par le prisme du système agraire. Cahiers Agricultures 24:224-31. doi: 10.1684/agr.2015.0758

Cialdella N, 2015. Do political changes aimed at reducing Amazonian deforestation contribute to ecological intensification? Cahiers Agricultures 24:246-54. doi: 10.1684/agr.2015.0761

FAO, 2014. Legacy of IYFF 2014 and the way forward. Rome: Food and Agriculture Organization of the United Nations. http://www.fao.org/3/ b-mm296e.pdf

Machum S, 2015. Shifting practices and shifting discourses: policy and small-scale agriculture in Canada. Cahiers Agricultures 24:232-9. doi: 10.1684/agr.2015.0756

Parrot L, Nantchouang A, Dongmo C, 2015. Les transformations de l'agriculture familiale dans les petites villes : le cas de Muea au Cameroun. Cahiers Agricultures 24:215-23. doi: 10.1684/agr.2015.0759

Saravia-Matus S, Gomez y Paloma S, 2015. Challenges in implementing the National Sustainable Agriculture Development Plan (NSADP) for subsistence and semisubsistence farmers in Sierra Leone. Cahiers Agricultures 24:240-5. doi: 10.1684/agr.2015.0757

Sourisseau JM, Kahane R, Fabre P, Hubert B, 2015. Actes des Rencontres internationales. Montpellier, France, 1-3 juin 2014. Montpellier: Agropolis international. http://www.agropolis.fr/pdf/publications/actes-rencontres-internationales-agriculturesfamiliales-recherche-juin-2014.pdf 